\title{
La medida de la capacidad reflexiva: instrumentos disponibles en castellano y tareas pendientes.
}

The measurement of reflective capacity: instruments available in spanish and pending tasks.

\author{
María Sánchez Pascual a, Leticia León Quismondo ${ }^{\text {b }}$, Araceli García López de Arenosa ${ }^{\text {c }}$, Josefina
}

Mas Hesse d, Alberto Fernández Liria e

${ }^{a, b, c, d y e}$ Hospital Universitario Príncipe de Asturias, Alcalá de Henares, Madrid, España.

Correspondencia: Alberto Fernández Liria (afiria@gmail.com)

Recibido: 01/12/2014; aceptado: 25/03/2015

RESUMEN: Introducción: La medida de la función reflexiva se ha convertido en un aspecto central para la evaluación e investigación de procesos en psicoterapia. Material y método: Se realizó una revisión sistemática de los instrumentos propuestos para la medida de la función reflexiva, actualizando la realizada por Luyten en el año 2011. Se realizó también una revisión de los instrumentos validados en lengua castellana. Resultados: Se encontraron cuestionarios, entrevistas, pruebas experimentales y proyectivas indicadas para la medida de función reflexiva. Conclusiones: En el momento actual faltan instrumentos validados en castellano para poder realizar investigaciones en este campo. Por esta razón, se hacen recomendaciones sobre formación de evaluadores, la puesta a prueba de instrumentos ya validados en muestras clínicas y la validación de instrumentos no disponibles en castellano.

PALABRAS CLAVE: test, mentalización, función reflexiva, escala, entrevista, cuestionario, medida.
ABSTRACT: Introduction: The measurement of reflective functioning has become a central aspect for evaluation and processes investigation in psychotherapy. Material and method: A systematic review of the proposed instruments for measuring reflective functioning was performed, updating that one realized by Luyten in 2011. A review of validated instruments in Spanish was also performed. Results: Questionnaires, interviews, experimental and projective tests indicated for the measurement of reflective functioning were found. Conclusions: At the current time, instruments validated in Spanish are absent to be able to perform investigations in this field. For this reason, recommendations for evaluators training, testing validated instruments in clinical samples and validation of non-available instruments in Spanish are performed.

KEY WORDS: test, mentalizing, mentalization, reflective functioning, scale, interview, questionnaire, measure.

\section{Introducción}

En los últimos años se han desarrollado intervenciones psicoterapéuticas que han demostrado su eficacia sobre distintas condiciones clínicas y que se han concebido con la intención declarada de potenciar la capacidad de mentalizar (1-9). Estas terapias "basadas en la mentalización" encuentran su base teórica en la teoría del apego propuesta por John Bowlby (10-14), en los desarrollos experimentales que de la misma han hecho autores como Ainsworth, Main y Fonagy (15-16), en la Teoría de la Mente desarrollada por Premack y Woodruff (17), y en los estudios realizados sobre la teoría de la mente en personas con autismo (18-19), con esquizofrenia (2021) y con personalidad límite (2). 
Para Bateman y Fonagy (22) la mentalización es un proceso básico para la regulación emocional, el funcionamiento interpersonal y el desarrollo de una identidad integrada. Las primeras propuestas de estos autores se dirigieron al tratamiento de las personas con trastorno límite de la personalidad, cuyas manifestaciones consideraban que podían entenderse como resultado de fallos en la mentalización.

Hoy ese grupo plantea que la mentalización puede ser considerada como una suerte de factor común en psicoterapia y el uso de intervenciones orientadas a promover la capacidad de mentalización han sido propuestas para otros muchos trastornos (2-9, 23-25).

Para este grupo de autores la capacidad de mentalización es algo que se puede detectar y medir, pudiendo ser utilizada como medida para guiar el tratamiento. Los primeros instrumentos empleados para medirla eran sumamente complicados y requerían de análisis formales del discurso sobre entrevistas estructuradas, como el Adult Attachment Interview (AAI) (26). Posteriormente se ha trabajado intensamente $-\mathrm{y}$ se sigue trabajando hoy - para desarrollar instrumentos de manejo más sencillo, aunque no se ha llegado a un acuerdo sobre la validez de ninguno de ellos que pueda ser considerado como un gold standard del modo en el que pueda serlo el original análisis de las narrativas del AAI (27). Pocos de los instrumentos desarrollados han sido validados y aún menos están disponibles en castellano.

Este trabajo pretende revisar los instrumentos propuestos para medir la capacidad reflexiva y, en base a ello, hacer una propuesta de trabajo para validar los necesarios para investigar en esta materia en castellano. Para ello tomaremos como base el capítulo de Luyten (27) donde se revisa el estado de la cuestión, incluyendo información sobre alguno de los que están en elaboración en el año 2014.

\section{Conceptos básicos}

Hay una serie de conceptos procedentes de diferentes campos de investigación que parecen referirse a las mismas funciones y que, a veces, han sido utilizados como sinónimos.

El concepto de capacidad reflexiva, que Fonagy y sus colaboradores definen en 1991 (28) proviene de los estudios sobre transmisión intergeneracional del vínculo de apego que realizaron estos autores, usando la Entrevista del Apego Adulto y observando si los sujetos se referían o no a estados mentales cuando se les pedía que hablaran de experiencias relacionales de su infancia.

La capacidad reflexiva puede ser entendida como un conjunto de funciones psíquicas superiores que nos permiten identificar y comprender nuestros propios estados mentales y los de los otros. Capacidades que, a través de un mecanismo representa- 
cional e inferencial, permiten interpretar las intenciones, los deseos, sentimientos y creencias, propios y ajenas. Es por tanto una capacidad compleja que permite al ser humano distinguir entre la realidad interna y externa. Como consecuencia de la posesión de estas cualidades, podemos predecir y explicar la propia conducta y de los otros (29).

La capacidad de mentalizar o mentalización es la capacidad para comprender el propio comportamiento y el de otros a través de la identificación y atribución de estados mentales. Se trata de un concepto multidimensional que incluye tanto procesos como contenidos mentales (30). La mentalización requiere procesos tales como la simbolización, procesamiento y transformación de representaciones mentales, pensamiento y emociones. Engloba además procesos de distinta complejidad, desde entender un estado afectivo hasta realizar una narración autobiográfica (31).

Esta capacidad se desarrolla a lo largo de la historia vital de la persona, influenciada en gran medida por las relaciones de apego tempranas con los progenitores. En este sentido, la alta capacidad reflexiva de un niño correlaciona con un apego seguro con sus progenitores, que a su vez correlaciona con una elevada capacidad reflexiva de los mismos $(15,32)$.

Tanto la mentalización como la función reflexiva comparten la función de tomar conciencia y regular los estados mentales. En este artículo utilizaremos el término capacidad reflexiva englobando las funciones referentes a la mentalización, ya que desarrollar la capacidad de mentalizar es incrementar la capacidad reflexiva.

En los últimos tiempos se ha concedido una importancia creciente a cuatro polaridades de la mentalización, que para funcionar adecuadamente deben estar bien equilibradas en cada momento. Estas polaridades se refieren a: procesos focalizados en sí mismo o en el otro, procesos automáticos o controlados, procesos cognitivos o afectivos, y procesos basados en manifestaciones externas o en el mundo interno de la persona $(22,29,33-34)$.

- Polaridad entre mentalización focalizada en sí mismo VS focalizada en el otro.

La mentalización focalizada en uno mismo, con interés por los propios sentimientos, pensamientos, etc., así como por los cambios en el estado mental en distintos momentos o contextos, permite entender la propia subjetividad, dejando siempre un espacio para reconocer que no siempre tenemos pleno acceso a nuestro funcionamiento mental. Focalizar en uno mismo permite mentalizar la afectividad, es decir, reflexionar sobre las propias emociones, comprendiendo su significado para nosotros en cada contexto $(27,31)$

Por otra parte, la mentalización focalizada en el otro (con el que la persona interacciona o no), se refiere a la capacidad de prestar atención a los estados mentales del otro y comprenderlos, entendiendo que el otro tiene su propio mundo interno, sus ideas, intenciones, valores y emociones propias y diferenciadas. Dentro de esta capacidad es importante darse cuenta de que el mundo interno del otro es opaco y que por 
ello sólo podemos aproximarnos a él con inferencias e hipótesis, de forma que una mentalización efectiva no se confunda con una "realidad" a la que no tenemos acceso (25).

- Polaridad entre mentalización automática o implícita VS mentalización controlada o explícita.

La mentalización automática o implícita transcurre por una serie de procesos no reflexivos ni conscientes, tratándose de un proceso rápido, intuitivo, y que incluye juicios, sentimientos y pensamientos de los cuales es difícil dar cuenta.

En contraste, la mentalización controlada o explicita es consciente, simbolizada (verbalizada) y requiere atención, esfuerzo y reflexión. Requiere por tanto una intención de exploración de los procesos mentales propios y ajenos (15). Esta simbolización permite una distancia entre lo que se piensa y la "realidad", y con ello cierta flexibilidad en el sistema de pensamiento.

- Polaridad entre mentalización focalizada en procesos cognitivos VS focalizada en procesos afectivos.

La mentalización focalizada en lo cognitivo permite reflexionar sobre el funcionamiento mental, es decir, relacionar estados mentales con conductas, predecir comportamientos, reflexionar sobre los propios pensamientos, etc., basándonos en pensamientos, creencias e interpretaciones.

En el polo opuesto se encuentra la mentalización focalizada en lo afectivo, que implica necesariamente algún grado de experiencia afectiva, ya sea de uno mismo, por contactar con el mundo emocional y afectivo propio, o de los demás, al captar su estado afectivo. En este sentido, esta capacidad se relaciona con la intuición, la empatía automática, la resonancia afectiva o la sintonización emocional.

- Polaridad entre mentalización centrada en el mundo externo VS centrada en lo interno.

La mentalización focalizada en lo externo se refiere a la reflexión sobre características externas propias o ajenas, tales como el aspecto físico, el lenguaje no verbal, los cambios fisiológicos, la expresión de emociones, etc.

En contraste, la polaridad focalizada en lo interno se refiere a la mentalización sobre las experiencias subjetivas, internas, mentales o emocionales, del sí mismo o de los otros, como pueden ser la autorreflexión o la reflexión e inferencias sobre el funcionamiento mental de los demás.

Para mentalizar de manera efectiva es necesaria una flexibilidad entre estas polaridades, de forma que predomine un polo u otro según el contexto, por ejemplo, permitiendo tanto captar rápida e intuitivamente algo que está ocurriendo en la relación con alguien como poder pararse a pensar sobre esto, así como poder cuestionarlo y darse cuenta de cómo esta primera impresión ha estado influida por creencias o prejuicios que distorsionan la percepción, o, por el contrario, son ajustadas y suficientemente fiables. Lanza Castelli (31) da el siguiente ejemplo: 
ORIGINALES Y REVISIONES

"el proceso de inferir deliberadamente las creencias que determinan cierto comportamiento de otra persona, supone el predominio de los siguientes polos: proceso focalizado en el otro (en la polaridad "focalizados en sí mismo o en el otro"), proceso controlado (en la polaridad "procesos automáticos o controlados"), proceso cognitivo (en la polaridad "procesos cognitivos o afectivos"), basado en lo interno (en la polaridad "procesos basados en lo interno o en lo externo"). En cambio, el empatizar espontáneo, que incluye una sintonía no deliberada con el estado afectivo del otro a la vista de la expresión de sus emociones, implica el predominio del polo "focalizado en el otro", del polo "procesos automáticos", del polo "proceso afectivo", del polo "basado en lo externo"."

La cognición social puede definirse como el conjunto de procesos cognitivos y emocionales que están implicados en situaciones de interacción social. Se compone de procesos como percibir, procesar y evaluar estímulos que nos permiten construir una representación social de nuestro entorno (35).

En este trabajo se tiene en cuenta el constructo de cognición social por su proximidad e inclusión dentro de lo que entendemos por capacidad reflexiva. En los últimos años se ha investigado mucho en el campo de la cognición social, campo que ha demostrado tener un importante poder explicativo en personas con esquizofrenia (20-21, 36), en la asociación con síntomas negativos (37-38), síntomas de desorganización (39) y encontrándose resultados más controvertidos para la asociación con sintomatología paranoide (40-41). En este mismo sentido, se ha estudiado también la relación de la cognición social con el síndrome de Asperger (42) y con población normal en situaciones de estrés (43). En relación al estudio que nos compete, algunas pruebas descritas para la evaluación de la cognición social pueden ser útiles para evaluar facetas de la capacidad reflexiva, dada la similitud de los constructos inherentes.

\section{Próposito}

El propósito de este trabajo es revisar qué instrumentos existen validados en castellano y qué trabajos de investigación habría que poner en marcha para dotarnos de los necesarios para investigar en este campo.

\section{Material y Método}

Partimos de la revisión de Luyten que incluye tanto textos publicados como en elaboración hasta 2011 (27). En ella se revisan 86 trabajos que hacen referencia a 42 
instrumentos de los que se intentó obtener la versión en el idioma original e información sobre adaptaciones en castellano validadas o en curso.

Tabla 1.

Cuestionarios en inglés

\begin{tabular}{|c|c|c|c|c|c|c|c|c|}
\hline \multirow[b]{3}{*}{ Cuestionarios } & \multicolumn{8}{|c|}{ Dimensiones } \\
\hline & \multicolumn{2}{|c|}{ Sí mismo-Otros } & \multicolumn{2}{|c|}{ Cognitivo-Afectivo } & \multicolumn{2}{|c|}{ Interno-Externo } & \multicolumn{2}{|c|}{$\begin{array}{c}\text { Automático- } \\
\text { Controlado }\end{array}$} \\
\hline & Sí mismo & Otros & Cognitivo & Afectivo & Interno & Externo & $\begin{array}{c}\text { Automá- } \\
\text { tico }\end{array}$ & $\begin{array}{c}\text { Contro- } \\
\text { lado }\end{array}$ \\
\hline $\begin{array}{l}\text { Beliefs about Emotions } \\
\text { Scale } \\
\text { (Rimes \& Chalder, } \\
\text { 2010) }\end{array}$ & $X$ & $(\mathrm{X})$ & $\mathrm{X}$ & $\mathrm{X}$ & $\mathrm{X}$ & & & $\mathrm{X}$ \\
\hline $\begin{array}{l}\text { Toronto Alexithymia } \\
\text { Questionnaire } \\
\text { (Bagby, Parker y Taylor, } \\
\text { 1994) }\end{array}$ & $X$ & & $X$ & $\mathrm{X}$ & $\mathrm{X}$ & & & $\mathrm{X}$ \\
\hline $\begin{array}{l}\text { Kentucky Mindfulness } \\
\text { Scale-Describe and } \\
\text { Act with Awareness } \\
\text { subscales } \\
\text { (Baer, Smith y Allen, } \\
\text { 2004) }\end{array}$ & $X$ & & $X$ & $\mathrm{X}$ & $\mathrm{X}$ & & (X) & $\mathrm{X}$ \\
\hline $\begin{array}{l}\text { Mindful Attention } \\
\text { Awareness Scale } \\
\text { (MAAS) } \\
\text { (Brown y Ryan, 2003) }\end{array}$ & $\mathrm{X}$ & & $\mathrm{X}$ & $\mathrm{X}$ & $\mathrm{X}$ & & (X) & $\mathrm{X}$ \\
\hline $\begin{array}{l}\text { Levels of Emotional } \\
\text { Awareness Scale } \\
\text { (Lane, Quinlan, } \\
\text { Schwartz y Walker, } \\
\text { 1990) }\end{array}$ & $\mathrm{X}$ & $\mathrm{X}$ & $\mathrm{X}$ & $\mathrm{X}$ & $\mathrm{X}$ & & & $\mathrm{X}$ \\
\hline $\begin{array}{l}\text { Psychological } \\
\text { Mindedness Scale } \\
\text { (Shill y Lumbey, 2002) }\end{array}$ & $\mathrm{X}$ & $\mathrm{X}$ & $X$ & $\mathrm{X}$ & $\mathrm{X}$ & & & $\mathrm{X}$ \\
\hline $\begin{array}{l}\text { Interpersonal } \\
\text { Reactivity Index (IRI) } \\
\text { (Davis, 1983) }\end{array}$ & & $\mathrm{X}$ & $\mathrm{X}$ & $\mathrm{X}$ & $\mathrm{X}$ & & & $\mathrm{X}$ \\
\hline $\begin{array}{l}\text { Empathy Quotient } \\
\text { (EQ) } \\
\text { (Lawrence, Shaw, } \\
\text { Baker, Baron-Cohen y } \\
\text { David, 2004) }\end{array}$ & $X$ & $\mathrm{X}$ & $X$ & $\mathrm{X}$ & $\mathrm{X}$ & & (X) & $\mathrm{X}$ \\
\hline $\begin{array}{l}\text { Mayer-Salovey-Caruso } \\
\text { Emotional Intelligence } \\
\text { Test } \\
\text { (Salovey y Grewal, } \\
\text { 2005) }\end{array}$ & $\mathrm{X}$ & $\mathrm{X}$ & $X$ & $\mathrm{X}$ & $\mathrm{X}$ & $\mathrm{X}$ & $(\mathrm{X})$ & $\mathrm{X}$ \\
\hline $\begin{array}{l}\text { Reflective Functioning } \\
\text { Questionaire } \\
\text { (Fonagy y Ghinai, 2008) }\end{array}$ & $X$ & $\mathrm{X}$ & $X$ & $\mathrm{X}$ & $\mathrm{X}$ & $(\mathrm{X})$ & & $\mathrm{X}$ \\
\hline $\begin{array}{l}\text { Parental Reflective } \\
\text { Functioning } \\
\text { Questionnaire } \\
\text { (Luyten et al., 2009) }\end{array}$ & $X$ & X & $X$ & $\mathrm{X}$ & $X$ & $(\mathrm{X})$ & & $\mathrm{X}$ \\
\hline $\begin{array}{l}\text { Mentalization Stories } \\
\text { Test for adolescents } \\
\text { (Vrouva y Fonagy, } \\
\text { 2009) }\end{array}$ & & $\mathrm{X}$ & $\mathrm{X}$ & $\mathrm{X}$ & $\mathrm{X}$ & $(\mathrm{X})$ & & $\mathrm{X}$ \\
\hline
\end{tabular}


Tabla 2.

Entrevistas en inglés

\begin{tabular}{|c|c|c|c|c|c|c|c|c|}
\hline \multirow{3}{*}{$\begin{array}{l}\text { Entrevistas o sistemas } \\
\text { de codificación de de } \\
\text { narrativas }\end{array}$} & \multicolumn{8}{|c|}{ Dimensiones } \\
\hline & \multicolumn{2}{|c|}{ Sí mismo-Otros } & \multicolumn{2}{|c|}{\begin{tabular}{|l|} 
Cognitivo-Afectivo \\
\end{tabular}} & \multicolumn{2}{|c|}{ Interno-Externo } & \multicolumn{2}{|c|}{$\begin{array}{c}\text { Automático- } \\
\text { Controlado }\end{array}$} \\
\hline & Sí mismo & Otros & $\begin{array}{c}\text { Cogni- } \\
\text { tivo }\end{array}$ & \begin{tabular}{|l} 
Afectivo \\
\end{tabular} & Interno & Externo & $\begin{array}{c}\text { Automá- } \\
\text { tico }\end{array}$ & $\begin{array}{c}\text { Contro- } \\
\text { lado }\end{array}$ \\
\hline $\begin{array}{l}\text { Adult Attachment } \\
\text { Interview- RFS } \\
\text { (Fonagy, Target, } \\
\text { Steele y Steele, 1998) }\end{array}$ & $\mathrm{X}$ & $\mathrm{X}$ & $\mathrm{X}$ & $\mathrm{X}$ & $\mathrm{X}$ & (X) & $(\mathrm{X})$ & $\mathrm{X}$ \\
\hline $\begin{array}{l}\text { Parent Development } \\
\text { Interview- RFS } \\
\text { (Slade, Aber, Berger, } \\
\text { Bresgi y Kaplan, } \\
\text { 2002) }\end{array}$ & $\mathrm{X}$ & $\mathrm{X}$ & $\mathrm{X}$ & $\mathrm{X}$ & $\mathrm{X}$ & (X) & $(\mathrm{X})$ & $\mathrm{X}$ \\
\hline $\begin{array}{l}\text { Working Model of } \\
\text { the Child Interview- } \\
\text { RFS } \\
\text { (Grienenberger, Kelly } \\
\text { v Slade 2005) }\end{array}$ & $\mathrm{X}$ & $\mathrm{X}$ & $\mathrm{X}$ & $\mathrm{X}$ & $\mathrm{X}$ & (X) & $(\mathrm{X})$ & $\mathrm{X}$ \\
\hline $\begin{array}{l}\text { Toronto Structured } \\
\text { Interview for } \\
\text { Alexithimia } \\
\text { (Bagby, Taylor, } \\
\text { Parker y Dickens, } \\
\text { 2006) }\end{array}$ & $\mathrm{X}$ & & $\mathrm{X}$ & $\mathrm{X}$ & $\mathrm{X}$ & & (X) & $\mathrm{X}$ \\
\hline $\begin{array}{l}\text { Mental States } \\
\text { Measure and Grille } \\
\text { de I'élaboration } \\
\text { Verbale de I'Affect } \\
\text { (Bouchard et al., } \\
2008 \text { ) }\end{array}$ & $\mathrm{X}$ & $\mathrm{X}$ & $\mathrm{X}$ & $\mathrm{X}$ & $\mathrm{X}$ & $\mathrm{X}$ & $(\mathrm{X})$ & $\mathrm{X}$ \\
\hline $\begin{array}{l}\text { Metacognition } \\
\text { Assessment Scale } \\
\text { (Carcione et al., } \\
\text { 2007) }\end{array}$ & $\mathrm{X}$ & $\mathrm{X}$ & $\mathrm{X}$ & $\mathrm{X}$ & $\mathrm{X}$ & & (X) & $\mathrm{X}$ \\
\hline $\begin{array}{l}\text { Intentionality Scale } \\
\text { Hill, Fonagy, } \\
\text { Lancaster y Broyden, } \\
\text { 2007) }\end{array}$ & & $\mathrm{X}$ & $\mathrm{X}$ & $\mathrm{X}$ & $\mathrm{X}$ & (X) & (X) & $\mathrm{X}$ \\
\hline $\begin{array}{l}\text { Internatinal State } \\
\text { Lexicon } \\
\text { (Beeghly y Cicchetti, } \\
1994 \text { ) }\end{array}$ & $\mathrm{X}$ & $\mathrm{X}$ & $\mathrm{X}$ & $\mathrm{X}$ & $\mathrm{X}$ & & (X) & $\mathrm{X}$ \\
\hline $\begin{array}{l}\text { Child Attachment } \\
\text { Interview (CAI) } \\
\text { Protocol } \\
\text { (Shmueli-Goetz, } \\
\text { Target, Fonagy y } \\
\text { Datta, 2008) }\end{array}$ & $\mathrm{X}$ & $\mathrm{X}$ & $\mathrm{X}$ & $\mathrm{X}$ & $\mathrm{X}$ & & (X) & $\mathrm{X}$ \\
\hline
\end{tabular}


Tabla 3.

Tareas experimentales en inglés

\begin{tabular}{|c|c|c|c|c|c|c|c|c|}
\hline \multirow{3}{*}{$\begin{array}{l}\text { Tareas experimentales- } \\
\text { observacionales }\end{array}$} & \multicolumn{8}{|c|}{ Dimensiones } \\
\hline & \multicolumn{2}{|c|}{ Sí mismo-Otros } & \multicolumn{2}{|c|}{\begin{tabular}{|l|} 
Cognitivo-Afectivo \\
\end{tabular}} & \multicolumn{2}{|c|}{ Interno-Externo } & \multicolumn{2}{|c|}{$\begin{array}{l}\text { Automático- } \\
\text { Controlado }\end{array}$} \\
\hline & Sí mismo & Otros & $\begin{array}{c}\text { Cogni- } \\
\text { tivo }\end{array}$ & Afectivo & Interno & Externo & $\begin{array}{c}\text { Automá- } \\
\text { tico }\end{array}$ & $\begin{array}{c}\text { Contro- } \\
\text { lado }\end{array}$ \\
\hline $\begin{array}{l}\text { Reading the Mind in } \\
\text { the Eyes Test } \\
\text { (Baron-Cohen, } \\
\text { Wheelwright, Hill, } \\
\text { Raste y Plumb, 2001) }\end{array}$ & & $\mathrm{X}$ & $\mathrm{X}$ & $\mathrm{X}$ & & $\mathrm{X}$ & & $\mathrm{X}$ \\
\hline $\begin{array}{l}\text { Reading the Mind in } \\
\text { Voice Test } \\
\text { (Golan, Baron-Cohen, } \\
\text { Hill y Rutherford, } \\
\text { 2007) }\end{array}$ & & $\mathrm{X}$ & $\mathrm{X}$ & $\mathrm{X}$ & & $\mathrm{X}$ & & $\mathrm{X}$ \\
\hline $\begin{array}{l}\text { Reading the Mind in } \\
\text { Films Task } \\
\text { (Golan, Baron-Cohen } \\
\text { y Golan, 2008) }\end{array}$ & & $\mathrm{X}$ & $\mathrm{X}$ & $\mathrm{X}$ & $\mathrm{X}$ & $\mathrm{X}$ & & $\mathrm{X}$ \\
\hline $\begin{array}{l}\text { International } \\
\text { Affective Picture } \\
\text { System (IAPS) } \\
\text { (Lang, Bradley y } \\
\text { Cuthbert, 2008) }\end{array}$ & & $\mathrm{X}$ & $\mathrm{X}$ & $\mathrm{X}$ & & $\mathrm{X}$ & & $\mathrm{X}$ \\
\hline $\begin{array}{l}\text { NimStim Set of } \\
\text { Facial Expressions } \\
\text { (Tottenham et al., } \\
2009 \text { ) }\end{array}$ & & $\mathrm{X}$ & $\mathrm{X}$ & $\mathrm{X}$ & & $\mathrm{X}$ & & $\mathrm{X}$ \\
\hline $\begin{array}{l}\text { Face Morphs } \\
\text { (Bailey et al., 2008) }\end{array}$ & $\mathrm{X}$ & $\mathrm{X}$ & $\mathrm{X}$ & $\mathrm{X}$ & & $\mathrm{X}$ & (X) & $\mathrm{X}$ \\
\hline $\begin{array}{l}\text { Dynamic Body } \\
\text { Expressions } \\
\text { Pichon, de Gelder y } \\
\text { Grèzes }(2009)\end{array}$ & & $\mathrm{X}$ & $\mathrm{X}$ & $\mathrm{X}$ & & $\mathrm{X}$ & (X) & $\mathrm{X}$ \\
\hline $\begin{array}{l}\text { Electromyography of } \\
\text { facial mimicry } \\
\text { (Sonnby-Borgström y } \\
\text { Jönsson, 2004) }\end{array}$ & (X) & $\mathrm{X}$ & (X) & $\mathrm{X}$ & & $\mathrm{X}$ & $\mathrm{X}$ & \\
\hline $\begin{array}{l}\text { Affect labeling } \\
\text { (Lieberman et al., } \\
\text { 2007) }\end{array}$ & & $\mathrm{X}$ & $\mathrm{X}$ & $\mathrm{X}$ & & $\mathrm{X}$ & & $\mathrm{X}$ \\
\hline $\begin{array}{l}\text { Movie for the } \\
\text { Assessment of Social } \\
\text { Cognition (MASC) } \\
\text { (Dziobek, Fleck, } \\
\text { Kalbe et al., 2006) }\end{array}$ & & $\mathrm{X}$ & $\mathrm{X}$ & $\mathrm{X}$ & $\mathrm{X}$ & $\mathrm{X}$ & $(\mathrm{X})$ & $\mathrm{X}$ \\
\hline $\begin{array}{l}\text { Trust Task } \\
\text { (King-Casas et al., } \\
2008)\end{array}$ & (X) & $\mathrm{X}$ & $\mathrm{X}$ & $\mathrm{X}$ & $\mathrm{X}$ & & & $\mathrm{X}$ \\
\hline $\begin{array}{l}\text { Interoceptive } \\
\text { Sensitive } \\
\text { (Barrett, Quigley, } \\
\text { Bliss-Moreau y } \\
\text { Aronson, 2004) }\end{array}$ & $\mathrm{X}$ & $\mathrm{X}$ & $\mathrm{X}$ & $\mathrm{X}$ & $\mathrm{X}$ & & & $\mathrm{X}$ \\
\hline
\end{tabular}




\begin{tabular}{|c|c|c|c|c|c|c|c|c|}
\hline $\begin{array}{l}\text { Empathy for Pain in } \\
\text { Others } \\
\text { (Hein y Singer, 2008) }\end{array}$ & $(\mathrm{X})$ & $\mathrm{X}$ & $\mathrm{X}$ & $\mathrm{X}$ & & $\mathrm{X}$ & $\mathrm{X}$ & $\mathrm{X}$ \\
\hline $\begin{array}{l}\text { Manipulating Body } \\
\text { Consciusness } \\
\text { (Brass, Schmitt, } \\
\text { Splenger y Gergely, } \\
\text { 2007); (Lenggenhager, } \\
\text { Tadi, Metzinger y } \\
\text { Blanke, 2007) }\end{array}$ & $\mathrm{X}$ & $\mathrm{X}$ & $\mathrm{X}$ & $\mathrm{X}$ & $\mathrm{X}$ & $\mathrm{X}$ & $X$ & $\mathrm{X}$ \\
\hline $\begin{array}{l}\text { Animated Theory of } \\
\text { Mind Inventory for } \\
\text { Children } \\
\text { (Beaumont y } \\
\text { Sofronoff, 2008) }\end{array}$ & & $X$ & $\mathrm{X}$ & $\mathrm{X}$ & $\mathrm{X}$ & $\mathrm{X}$ & (X) & $X$ \\
\hline $\begin{array}{l}\text { Maternal Mind } \\
\text { Mindedness } \\
\text { (Meins y Fernyhough, } \\
\text { 2006) }\end{array}$ & $\mathrm{X}$ & $X$ & $\mathrm{X}$ & $\mathrm{X}$ & $\mathrm{X}$ & $(\mathrm{X})$ & $(\mathrm{X})$ & $\mathrm{X}$ \\
\hline $\begin{array}{l}\text { Maternal Accuracy } \\
\text { Paradigm } \\
\text { (Sharp, Fonagy y } \\
\text { Goodyer, 2006) }\end{array}$ & & $X$ & $\mathrm{X}$ & $\mathrm{X}$ & $\mathrm{X}$ & $(\mathrm{X})$ & (X) & $\mathrm{X}$ \\
\hline $\begin{array}{l}\text { Strange Stories Task } \\
\text { (Happé, 2004) }\end{array}$ & & $\mathrm{X}$ & $\mathrm{X}$ & $\mathrm{X}$ & $\mathrm{X}$ & & & $\mathrm{X}$ \\
\hline
\end{tabular}

Tabla 4.

Test proyectivos en inglés

\begin{tabular}{|l|c|c|c|c|c|c|c|c|}
\hline \multirow{2}{*}{ Test proyectivos } & \multicolumn{9}{|c|}{ Dimensiones } \\
\cline { 2 - 9 } & Sí mismo-Otros & \multicolumn{2}{c|}{ Cognitivo-Afectivo } & \multicolumn{2}{|c|}{ Interno-Externo } & \multicolumn{2}{c|}{$\begin{array}{c}\text { Automático-Con- } \\
\text { trolado }\end{array}$} \\
\cline { 2 - 9 } & Sí mismo & Otros & $\begin{array}{c}\text { Cogni- } \\
\text { tivo }\end{array}$ & Afectivo & Interno & Externo & $\begin{array}{c}\text { Automá- } \\
\text { tico }\end{array}$ & $\begin{array}{c}\text { Contro- } \\
\text { lado }\end{array}$ \\
\hline $\begin{array}{l}\text { Thematic Appercep- } \\
\text { tion Test } \\
\text { (Luyten et al., 2010) }\end{array}$ & $(\mathrm{X})$ & $\mathrm{X}$ & $\mathrm{X}$ & $\mathrm{X}$ & $\mathrm{X}$ & $\mathrm{X}$ & $\mathrm{X})$ & $\mathrm{X}$ \\
\hline $\begin{array}{l}\text { Projective Imagina- } \\
\text { tion Test } \\
\text { (Blackshaw, Kinder- } \\
\text { man, Hare y Hatton, } \\
\text { 2001) }\end{array}$ & $\mathrm{X})$ & $\mathrm{X}$ & $\mathrm{X}$ & $\mathrm{X}$ & $\mathrm{X}$ & $\mathrm{X}$ & $\mathrm{X})$ & $\mathrm{X}$ \\
\hline
\end{tabular}

Se hizo una búsqueda exhaustiva en Pubmed con la siguiente estrategia de búsqueda que se ha actualizado hasta 01/10/2014:

Revisión sistemática, Meta-análisis: (((Test*[Title]) AND (((mentalizing) OR mentalization) OR ((reflective*) AND ((functioning) OR function))))) OR $((((($ mentalizing[Title/Abstract] $) \quad$ OR mentalization[Title/Abstract])) OR ((reflective*[Title/Abstract]) AND (functioning OR function[Title/Abstract])))) AND ((((scale*) OR interview*)) OR ((questionnaire*) OR measure*)))

La búsqueda bibliográfica identificó 58 artículos más. Se consiguieron todos los artículos a texto completo, que se distribuyeron entre los miembros de los distintos subgrupos para su evaluación. 
Tabla 5.

Instrumentos en castellano

\begin{tabular}{|c|c|c|c|c|c|c|c|c|}
\hline \multirow[b]{3}{*}{ Cuestionarios } & \multicolumn{8}{|c|}{ Dimensiones } \\
\hline & \multicolumn{2}{|c|}{ Sí mismo-Otros } & \multicolumn{2}{|c|}{ Cognitivo-Afectivo } & \multicolumn{2}{|c|}{ Interno-Externo } & \multicolumn{2}{|c|}{$\begin{array}{c}\text { Automático-Con- } \\
\text { trolado }\end{array}$} \\
\hline & Sí mismo & Otros & $\begin{array}{c}\text { Cogni- } \\
\text { tivo }\end{array}$ & Afectivo & Interno & Externo & $\begin{array}{c}\text { Automá- } \\
\text { tico }\end{array}$ & $\begin{array}{c}\text { Contro- } \\
\text { lado }\end{array}$ \\
\hline $\begin{array}{l}\text { Escala de Alexitimia } \\
\text { de Toronto (Toronto } \\
\text { Alexithymia Ques- } \\
\text { tionnaire) } \\
\text { (Moral de la Rubia, } \\
\text { J., Retamales, R., } \\
\text { 2001) }\end{array}$ & $\mathrm{X}$ & & $\mathrm{X}$ & $\mathrm{X}$ & $\mathrm{X}$ & & & $\mathrm{X}$ \\
\hline $\begin{array}{l}\text { Spanish version of } \\
\text { Mindful Attention } \\
\text { Awareness Scale } \\
\text { (MAAS) } \\
\text { (Soler, J, Tejedor, } \\
\text { R., Feliu-Soler, A., } \\
\text { Pascual, J.C., Cebo- } \\
\text { lla, A., Soriano, J., } \\
\text { Álvarez, E., y Pérez, } \\
\text { V..., 2012) }\end{array}$ & $\mathrm{X}$ & & $\mathrm{X}$ & $\mathrm{X}$ & $\mathrm{X}$ & & (X) & $\mathrm{X}$ \\
\hline $\begin{array}{l}\text { Índice de reactivi- } \\
\text { dad Interpersonal } \\
\text { (IRI) } \\
\text { (Pérez-Albéniz, A., } \\
\text { de Paul, J., Etxebe- } \\
\text { rría, J, Paz, M., y } \\
\text { Torres, E., 2003) }\end{array}$ & $\mathrm{X}$ & $\mathrm{X}$ & $\mathrm{X}$ & $\mathrm{X}$ & $\mathrm{X}$ & & & $\mathrm{X}$ \\
\hline $\begin{array}{l}\text { Spanish version of } \\
\text { the Mayer-Salovey- } \\
\text { Caruso Emotional } \\
\text { Intelligence Test } \\
\text { (MSCEIT) } \\
\text { (Extremera, N., } \\
\text { Fernández-Berroal, P. } \\
\text { y Salovey, P., 2006) }\end{array}$ & $\mathrm{X}$ & $\mathrm{X}$ & $\mathrm{X}$ & $\mathrm{X}$ & $\mathrm{X}$ & $\mathrm{X}$ & $(\mathrm{X})$ & $\mathrm{X}$ \\
\hline $\begin{array}{l}\text { Método para la } \\
\text { Evaluación de la } \\
\text { Mentalización en el } \\
\text { Contexto Interper- } \\
\text { sonal (MEMCI) } \\
\text { (Lanza Castelli, G. y } \\
\text { Bilbao, I., 2011) }\end{array}$ & $\mathrm{X}$ & $\mathrm{X}$ & $\mathrm{X}$ & $\mathrm{X}$ & $\mathrm{X}$ & $\mathrm{X}$ & $\mathrm{X}$ & $\mathrm{X}$ \\
\hline $\begin{array}{l}\text { Test de Situaciones } \\
\text { para la Evaluación } \\
\text { de la Mentalización } \\
\text { (TESEM) } \\
\text { (Lanza Castelli, G., } \\
\text { 2010) }\end{array}$ & $\mathrm{X}$ & $\mathrm{X}$ & $\mathrm{X}$ & $\mathrm{X}$ & $\mathrm{X}$ & $\mathrm{X}$ & (X) & $\mathrm{X}$ \\
\hline
\end{tabular}


ORIGINALES Y REVISIONES

\begin{tabular}{|c|c|c|c|c|c|c|c|c|}
\hline \multirow{3}{*}{$\begin{array}{l}\text { Tareas experimenta- } \\
\text { les-observacionales }\end{array}$} & \multicolumn{8}{|c|}{ Dimensiones } \\
\hline & \multicolumn{2}{|c|}{ Sí mismo-Otros } & \multicolumn{2}{|c|}{ Cognitivo-Afectivo } & \multicolumn{2}{|c|}{ Interno-Externo } & \multicolumn{2}{|c|}{\begin{tabular}{|c|}
$\begin{array}{c}\text { Automático-Con- } \\
\text { trolado }\end{array}$ \\
\end{tabular}} \\
\hline & Sí mismo & Otros & Cognitivo & Afectivo & Interno & Externo & $\begin{array}{c}\text { Automá- } \\
\text { tico }\end{array}$ & $\begin{array}{l}\text { Contro- } \\
\text { lado }\end{array}$ \\
\hline $\begin{array}{l}\text { Test de "La lectura } \\
\text { de la mente en los } \\
\text { ojos" } \\
\text { (Fernández-Abascal, } \\
\text { E., Cabello, R., } \\
\text { Fernández-Berrocal, } \\
\text { P. y Baron Cohen, } \\
\text { S., 2013) }\end{array}$ & $\mathrm{X}$ & $\mathrm{X}$ & $\mathrm{X}$ & $\mathrm{X}$ & $\mathrm{X}$ & $\mathrm{X}$ & $\mathrm{X}$ & $\mathrm{X}$ \\
\hline $\begin{array}{l}\text { Spanish version of } \\
\text { Movie for the As- } \\
\text { sessment of Social } \\
\text { Cognition (MASC) } \\
\text { (Lahera, G. et al., } \\
\text { 2014) }\end{array}$ & & $\mathrm{X}$ & $\mathrm{X}$ & $\mathrm{X}$ & $\mathrm{X}$ & $\mathrm{X}$ & (X) & $\mathrm{X}$ \\
\hline
\end{tabular}

\section{Resultados}

Instrumentos existentes en inglés

En el trabajo de Luyten (27) se revisan los instrumentos que se detallan en la tabla 1 clasificados en tres categorías:

1) cuestionarios

2) entrevistas o sistemas de codificación de narrativas

3) tareas experimentales

En relación a los cuestionarios (Tabla 1), ninguno de ellos mide el funcionamiento en todas las polaridades. El que más se aproxima a ello es el Mayer-SaloveyCaruso Emotional Intelligence Test (44), aunque los autores reconocen que no mide satisfactoriamente la mentalización automática. Ésta sólo es medida (nunca satisfactoriamente a juicio de Luyten y colaboradores), por el Kentucky Mindfulness ScaleDescribe and Act with Awareness subscales (45), el Mindful Attention Awareness Scale (46) y el Empathy Quotient (47), ninguno de los cuales atiende a la mentalización externa. De ésta sólo se ocupan (y sólo parcialmente), además del ya citado Mayer-Salovey-Caruso Emotional Intelligence Test (44), el Reflective Functioning Questionnaire (48), el Parental Reflective Functioning Questionnaire (49), Mentalization Stories Test for Adolescents (50).

Luyten (27) refleja el acuerdo existente en que las entrevistas o análisis de narrativas son las únicas pruebas que poseen una alta fiabilidad y validez. Una de las principales limitaciones que presentan estas pruebas son la gran cantidad de tiempo que ha de emplearse en la aplicación y la extensa formación que requieren los investigadores para lograr una interfiabilidad entre observadores adecuada. Luyten señala también como una limitación a tener en cuenta, el carácter retrospectivo de 
su corrección y su baja generalización debido a la especificidad de su contenido (relaciones interpersonales específicas). Para subsanar el carácter retrospectivo de las pruebas sugiere la posibilidad de trabajar con pruebas donde se pueda medir dicha capacidad de manera inmediata (situaciones de estrés, procedimiento de procesamiento afectivo, medidas de movimientos oculares o EEG).

Con respecto a tareas experimentales Luyten (27) señala su capacidad para poder medir de manera diferenciada e integrada aspectos cognitivos y afectivos. Estas pruebas han demostrado ser útiles para medir diferentes problemas en la mentalización y son notablemente menos costosas de aplicar que el análisis de narrativas (y, aunque más que los cuestionarios, son menos tediosas que éstos). Un ejemplo de ellas es The Reading the Mind in the eyes (51) que mide la integración de aspectos cognitivos y afectivos. Existen otras pruebas que lo hacen de manera diferenciada como Empathy Quotient de Baron-Cohen (47).

No han podido ser obtenidos los artículos de los siguientes instrumentos: Reflective Functioning Questionnaire (48), Parental Reflective Functioning Questionnaire (49), Mentalization Stories Test for Adolescents (50), Parent Development Interview (52), Mental States Measure and Grille de l'élaboration Verbale de l'Affect (53), Metacognition Assessment Scale (54), Internatinal State Lexicon (55), Trust Task (56), Animated Theory of Mind Inventory for Children (57), Maternal Mind Mindedness (58), Maternal Accuracy Paradigm y Thematic Apperception Test (59).

\section{Instrumentos en Castellano} bla 5):

Se han encontrado versiones en castellano de los siguientes instrumentos (Ta-

- Test de Situaciones para la Evaluación de la Mentalización (TESEM) (60). Es un instrumento en donde se le presentan una serie de situaciones al sujeto sobre las cuales se realizan preguntas que promueven la capacidad reflexiva. Las respuestas del entrevistado son posteriormente tabuladas, y puntuadas, de modo tal que se realizan dos tipos de análisis, cuantitativo y cualitativo. El análisis cuantitativo se basa en la Escala para la Función Reflexiva (61), con algunos agregados. Evalúa todas las dimensiones que componen la capacidad reflexiva desde el modelo de polaridades.

- Método para la Evaluación de la Mentalización en el Contexto Interpersonal (MEMCI) (31). Es un instrumento que puede aplicarse a través de dos procedimientos distintos: Solicitar al sujeto que relate episodios vinculares con personas significativas de su medio social o familiar. También a través de la entrevista RAP (Relationship Anecdotes Paradigm), una entrevista estructurada en la que se pide que relate diez episodios relacionales específicos en los que describa algo que ocurrió con otra persona, indicando cuándo y con quién tuvo lugar dicho episodio, así como lo que la otra persona y él mismo hicieron o dijeron. 
A medida que el paciente o entrevistado relata cada uno de los episodios relacionales mencionados, se procede a formularle las preguntas específicas para evaluar distintos aspectos de la mentalización presentes en dicho episodio.

- Mesuring Emotional Intelligence with the MSCEIT V2.0 (Versión Española) (62). Evalúa percepción de emociones con precisión, el uso de emoción para facilitar pensamiento, emoción comprensiva, y la gestión de emociones. Las tareas que se proponen en la aplicación y el modo de corrección son semejantes a la versión original. Está pendiente la realización de más estudios que refuercen la validez de la prueba.

- Movie for the Assessment of Social Cognition (MASC) (Versión Española) (63). Consiste en un método audiovisual de evaluación de la cognición social, en el que el sujeto debe realizar una serie de inferencias acerca de los estados mentales de los protagonistas de la historia. Se muestra un vídeo de 15 minutos en el que aparecen 4 personajes interaccionando. El vídeo se detiene en 46 ocasiones, preguntando al sujeto acerca de los sentimientos, pensamientos e intenciones de sus protagonistas. A través de este procedimiento se obtiene una puntuación total en cognición social y en los tres tipos de errores (hipermentalización, hipomentalización y no mentalización).

- Mindful Atention Awareness Scale (MAAS) (Versión Española) (64). Es un autoinforme formado por 15 ítems de escala tipo likert que evalúa la capacidad general de mindfulness de un individuo. La adaptación española goza de buenas propiedades, tanto en términos de validez como de fiabilidad.

- Índice de Reactividad Interpersonal (IRI) (65). Al igual que en la versión original, nace como una escala para evaluar la empatía desde una aproximación multidimensional. Se trata de un cuestionario de 28 ítems divididos en 4 subescalas (Toma de perspectiva, Fantasía, Preocupación empática y Estrés interpersonal). En concreto, este instrumento evalúa la empatía implícita (que tiene que ver con lo necesario para poner en marcha la mentalización implícita).

- Test de lectura de la mente en los ojos (66). Este instrumento está traducido al castellano pero no está validado actualmente. Cuenta con 36 fotografías de la región de los ojos, en las que la persona tiene que elegir qué está pensando o sintiendo la persona, con 4 opciones de respuesta. El instrumento incluye un glosario de términos por si no estás seguro de las palabras que aparecen entre las opciones de respuesta.

- Cuestionario de Alexitimia de Toronto (Toronto Alexithymia Questionnaire (TAS20) (67). Se trata de una escala autoaplicada de 20 ítems con seis posibilidades de respuesta, que van desde "muy en desacuerdo" hasta "muy de acuerdo" y que se puntúan entre 0 y 5 . Consta de tres factores: dificultad en identificar sentimientos, dificultad para describir sentimientos y pensamiento orientado externamente. Este instrumento está orientado a detectar la capacidad del sujeto para identificar las 
emociones propias y darles expresión verbal y ha sido muy utilizado en trastornos del espectro autista. Ha sido validado en varias poblaciones además de en la española, como en la griega y la italiana.

\section{Discusión}

\section{Entrevistas}

El patrón oro para la evaluación de la capacidad reflexiva son las entrevistas semiestructuradas que se evalúan mediante la aplicación de unas escalas que valoran la cualidad del discurso (27). Estas incluyen la historia de paciente, su historia de relaciones de apego pasada y actual y evaluación concreta de las quejas de los pacientes y los síntomas que estén relacionados con estas relaciones.

Existen dos de ellas que miden todas las dimensiones de la capacidad reflexiva propuestas por Luyten. La Adult Attachment Interview-RFS (61) mide la representación del apego adulto a través de los recuerdos de la infancia de la persona mediante el análisis del discurso. Es un instrumento con buenas propiedades psicométricas que está validado en diversas muestras y que, además, incluye una escala que evalúa la capacidad de mentalización del individuo. Sin embargo, presenta como inconveniente que requiere un proceso laborioso de formación del profesional para su administración y calificación. La Working Model of the Child Interview-RFS (68), evalúa la representación interna que el adulto tiene con su hijo. Tiene la ventaja de que puede administrarse de manera autoaplicada o en vídeo, lo que supondría un ahorro de tiempo importante.

Por otro lado, existe una versión para población infantil entre los 7 y los 12 años con características parecidas a la Adult Attachment Interview-RFS (61), la Child Attachment Interview (CAI) Protocol (69). En esta entrevista se solicita a la persona una descripción de la relación con su cuidador principal. Este instrumento tiene el objetivo de valorar la representación propia y de los otros y que posee buenas propiedades psicométricas.

Otra entrevista disponibles para la evaluación de la función reflexiva, no tan completa como las anteriores, es la Toronto Structured Interview for Alexithimia (70). $\mathrm{Al}$ igual que su correspondiente versión en formato cuestionario, el Toronto Alexithymia Questionnaire (71), no atiende a la dimensión interpersonal, sino que únicamente evalúa la capacidad para identificar sentimientos y describirlos.

\section{Cuestionarios}

De los cuestionarios disponibles, el MSCEIT (44), el Reflective Functioning Questionarie (48) y el Parental Reflective Functioning Questionarie (49) evalúan 
ORIGINALES Y REVISIONES

todas las dimensiones que conforman la capacidad reflexiva a excepción del polo automático.

El MSCEIT (44) es el único que evalúa todas las dimensiones de la capacidad reflexiva. Existe una adaptación al castellano (62) y la posibilidad de ser administrado por ordenador con los beneficios de costes que eso conlleva. Como limitaciones, que en el propio estudio señalan, existe la necesidad de estudios posteriores que valoren su validez y medida de distintas variables.

El RFQ (Reflective Functioning Questionarie) (48) y el PRFQ (Parental Reflective Functioning Questionarie) (49) también miden todas las esferas de la capacidad reflexiva. Aunque aparecen citados en el capítulo de Luyten (27) se trata de manuales no publicados todavía porque están en desarrollo y tampoco están publicadas sus propiedades psicométricas por lo que no podemos realizar un análisis exhaustivo de los mismos.

Otro grupo de cuestionarios que miden casi todas las esferas que conforman la capacidad reflexiva, excepto lo referente al mundo externo y el funcionamiento automático, son Levels of Emotional Awareness Scale (72), Psychological Mindedness Scale (73), Empathy Quotient (EQ) (47) y Beliefs about Emotions Scale (74).

El Levels of Emotional Awareness Scale (72) está más destinado a la medida de conciencia emocional, no mide todas las áreas que conforman la funciones reflexiva omitiendo aspectos importantes como las intenciones. Requiere capacidad de identificación y expresión emocional por parte de los sujetos a quienes se les administra por lo que limita el área de aplicación muestral. Por parte del equipo experto requiere un entrenamiento específico en la aplicación y la corrección del mismo.

El Psychological Mindedness Scale (73) sí que evalúa la capacidad del individuo para reflexionar sobre pensamientos y sentimientos así como acciones e intenciones de sí mismo y de los otros.

El Beliefs about Emotions Scale (74) únicamente proporciona información sobre las creencias del individuo acerca de sus propias emociones pero no mide el componente interpersonal de las mismas. Entre sus ventajas se encuentran su sencilla aplicación y sus buenas propiedades psicométricas, el inconveniente es que dichas propiedades están limitadas al estudio de una patología concreta, la fatiga crónica, sin grupo control de comparación.

El Empathy Quotient (47) es el único de este grupo que evalúa la esfera automática de la capacidad reflexiva. Apoya la perspectiva multidisciplinar de la empatía, tanto cognitiva, emocional como de las habilidades sociales. Las ventajas que presenta es que atiende a todas las dimensiones mencionadas anteriormente a excepción de la valoración mundo externo. Es de fácil aplicación y está validado. Además está basado en la teoría empatía de Baron Cohen que presenta un gran apoyo empírico.

Los cuestionarios que no evalúan la dimensión interpersonal y por tanto el mundo externo del sujeto son Mindful Attention Awareness Scale (MAAS) (46) y 
ORIGINALES Y REVISIONES

Kentucky Mindfulness Scale-Describe and Act with Awareness subscales (45).

\section{Tareas Experimentales}

Han sido muy diversas las estrategias utilizadas para evaluar la función reflexiva mediante tareas experimentales. Estas pruebas han demostrado ser útiles para medir de manera diferenciada e integrada tantos los aspectos cognitivos como los afectivos de la metacognición. Una ventaja global de este tercer grupo de instrumentos es que resultan menos costosos en su aplicación en comparación con las entrevistas, así como menos tediosos que los cuestionarios.

De las tareas examinadas, la única que evalúa todas las dimensiones propuestas de la capacidad reflexiva es la Manipulating Body Consciusness (75), siendo un instrumento que hace hincapié en la capacidad del individuo para predecir las intenciones de los otros en una variedad de contextos.

Existe un grupo de instrumentos que evalúa las dimensiones de la capacidad reflexiva que tienen que ver con el los otros, con el mundo externo y con el funcionamiento controlado. En este grupo se encontrarían las siguientes pruebas: Reading the Mind in the Eyes Test (51), Reading the Mind in Voice Test (76), NimStim Set of Facial Expressions (77), Affect Labelling (78). El Reading the Mind in Films Task (79) se diferencia de los anteriores en que sí evalúa el mundo interno de la persona.

El grupo de trabajo de Baron Cohen ha elaborado el Reading the Mind in the Eyes Test (51), el Reading the Mind in Voice Test (76) y el Reading the Mind in Films Task (79), los cuales han ido adquiriendo repercursión en su uso como medida mind reading. Cabría destacar el Reading the Mind in Films Task (79), en el que al participante se le muestran 22 escenas sociales breves en formato de película. Requiere una integración multimodal de la información socio-emocional y, por tanto, añade validez ecológica a los anteriores instrumentos. Además, las puntuaciones en esta escala correlacionan significativamente con las tareas complejas de reconocimiento de emociones, lo que confirma su validez, tales como The Cambridge Mindreading Face-Voice Battery (CAM) (80).

Similar a la anterior, aparece la prueba Movie for the Assessment of Social Cognition (MASC) (42) que evalúa todas las dimensiones de la capacidad reflexiva, excepto la relacionada con el sí mismo. En ella, el participante tiene que visionar una película en la que interaccionan cuatro personajes, y por tanto se trataría de mentalización en tercera persona. No obstante, podría tenerse en cuenta cómo influye el proceso de identificación con los personajes en la elección de las respuestas. No implica al observador como agente en la interacción, por lo que puede haber aspectos que comprometen la capacidad de mentalización que no estén contemplados por la prueba. La prueba proporciona una puntuación global en cognición social, además de puntuaciones en los errores específicos de esta capacidad (errores por hipermentalización, por hipomentalización y de no mentalización). A pesar de que su tiempo de 
administración es extenso (aproximadamente una hora) muestra la ventaja de poder ser autoaplicado o administrado en grupo, y de presentar un uso extenso en poblaciones con limitación en la capacidad de mind reading. El instrumento ha sido traducido y validado en castellano por nuestro grupo (63).

Por su parte, el NimStim Set of Facial Expressions (77) queda restringido a la medida del reconocimiento expresivo de rostros, al igual que Reading the Mind in the Eyes Test (51), mientras que el Affect Labelling (78) añade la medida del procesamiento no lingüístico del afecto.

Existe otra vertiente de instrumentos elaborados que evalúan la respuesta fisiológica cerebral del individuo ante estímulos emocionales, aunque no han sido utilizados en poblaciones clínicas. Entre estos se encontraría el Electromyography of Facial Mimicry (81) Dynamic Body Expressions (82), Face Morphs (83) y Empathy for Pain in Others (84).

El estudio de la pérdida de la capacidad reflexiva en situaciones de interacción social, situaciones bajo tensión, supondría una línea de investigación de interés. Para ello sería necesaria la elaboración de autoinfomes accesibles y poco complejos que den cuenta de ello. Actualmente se puede obtener un acercamiento a este tipo de medida desde paradigmas de pruebas experimentales pero todavía presentan limitaciones en cuanto a que requieren tecnologías muy complejas y costosas de aplicar (27).

Los instrumentos desarrollados en Argentina por Lanza Castelli descritos más arriba, Test de Situaciones para la Evaluación de la Mentalización (TESEM) (60) y Método para la Evaluación de la Mentalización en el Contexto Interpersonal (ME$M C I)$ (31) han sido poco utilizados en población clínica y son de aplicación complicada, aunque parecen prometedores.

\section{Proyectivos}

El Projective Imagination Test (85) mide todas las dimensiones de la capacidad reflexiva de manera adecuada y exhaustiva y ha sido utilizado con varias poblaciones. La prueba consiste en la presentación de cuatro fotografías con escenas ambigüas, y a partir de las cuales al sujeto se le realizan preguntas sin claves ("¿Qué está pensando en esta imagen?") o preguntas con claves ("¿Qué crees que podría estar pensando o sintiendo el personaje?"), así como que describa con sus propias palabras la historia que muestra el dibujo.

\section{A modo de conclusión}

Como hemos señalado anteriormente, el principal problema para la medida de la función reflexiva es que los instrumentos cuya validez se ha demostrado de modo 
concluyente (los que se basan en el análisis de narrativas producidas sobre entrevistas más o menos estructuradas u otros facilitadores más o menos estructurados de producción de discurso, como las pruebas proyectivas) son de aplicación e interpretación trabajosa difícil, tediosa y que sólo es posible tras procesos largos y complejos de formación de los investigadores. La aplicación de análisis sistemáticos del discurso a diferentes materiales, especialmente al Adult Attachment Interview (61), pero también a otros materiales como Child Attachament Interview (69) y Object Relations Inventory (86), sustentan la pretendida validez del constructo de capacidad reflexiva. Estas pruebas tienen varios inconvenientes: son muy costosas y complejas de pasar, la interfiabilidad entre observadores sólo puede garantizarse a través de procesos de formación muy complejos y bajo la supervisión.

Recientemente se han desarrollado instrumentos tales como la escala de Función Reflexiva (61) y programas de entrenamiento (como los que el grupo de Bateman y Fonagy llevan a cabo en la Fundación Anna Freud), que permiten formar investigadores para analizar de manera específica la capacidad reflexiva y no otros aspectos que pueden ser medidos desde el AAI, haciendo más aplicables estas estrategias.

Existen distintas líneas de investigación vinculadas al desarrollo de análisis de narrativas y transcripciones relacionados con la medida de capacidad reflexiva. Actualmente la validación del auto informe basado en el Cuestionario de Función Reflexiva (48) está en vías investigación.

La mayoría de los instrumentos evalúan el polo de capacidad reflexiva controlada aunque existen excepciones, como la Adult Attachment Interview-RFS (61) y el Reflective Functioning Questionarie (48), que incluyen la evaluación del polo automático. Para el desarrollo de la medida del polo automático, estos instrumentos pueden ser aplicados de manera concurrente con situaciones que evoquen tensión, procedimientos afectivos priming ${ }^{1}$, el rastreo de ojo o EEG que aportan información sobre procesos menos controlados de la mentalización y permite valorar la automatización de la capacidad reflexiva teniendo en cuenta sus variantes, incidiendo en la baja mentalización presente en varios trastornos.

Las tablas incluidas en el presente artículo proporcionan una visión general de diferentes instrumentos que pueden ser adaptados para la medida de capacidad reflexiva en diferentes poblaciones y patologías. Dichos instrumentos evalúan de manera diferenciada los polos que componen la capacidad reflexiva.

A la vista de lo propuesto por Luyten (27) y la revisión que ha sido realizada, teniendo en cuenta los diferentes aspectos subyacentes a la capacidad reflexiva, podría tener interés:

El Priming Afectivo es un procedimiento conductual en la que se mide el tiempo de reacción de un participante en una tarea donde se reflejan las preferencias implícitas sin que el participante pueda manipular voluntariamente la respuesta. Fazio et al. (1986). 
ORIGINALES Y REVISIONES

- Formar y acreditar evaluadores capaces de trabajar con las entrevistas semiestructuradas (AAI) (61) en castellano para poder utilizarlas como Gold Standar.

- Poner a prueba en poblaciones clínicas algunos instrumentos validados en castellano:

o Mesuring Emotional Intelligence with the MSCEIT V2.0 (Versión Española) (62).

o Movie for the Assessment of Social Cognition (MASC) (Versión Española) (63).

- Test de Situaciones para la Evaluación de la Mentalización (TESEM) (60).

- Método para la Evaluación de la Mentalización en el Contexto Interpersonal (MEMCI) (31).

- Validar en castellano los instrumentos más completos de los que ofertan facilidad de aplicación:

o The Reading the Mind in the eyes (51).

o Metacognition Assessment Scale (54).

- Contactar con los grupos que están desarrollando instrumentos prometedores para intentar un desarrollo paralelo de versiones en castellano:

o Cuestionario de función reflexiva (48).

o Test de Situaciones para la Evaluación de la Mentalización (TESEM) (60).

o Método para la Evaluación de la Mentalización en el Contexto Interpersonal (MEMCI) (31).

Una de las dificultades para llevar a cabo este trabajo consiste en que el patrón de oro para la validación debería ser la interpretación de entrevistas y, por tanto sería fundamental contar con un panel de evaluadores de éstas debidamente capacitados para realizar esta función.

\section{BIBLIOGRAFÍA}

(1) Bateman AW, Fonagy P. Individual Techniques of the Basic Model. In: Bateman AW, Fonagy P, editors. Handbook of Mentalizing in Mental Health Practice. American Psychiatric Publishing, Inc.; 2012. p. 67-80.

(2) Bateman AW, Fonagy P. Borderline Personality Disorder. In: Bateman AW, Fonagy P, editors. Handbook of Mentalizing in Mental Health Practice. American Psychiatric Publishing, Inc.; 2012. p. 273-88.

(3) Bateman AW, Fonagy P. Antisocial Personality Disorder. In: Bateman AW, Fonagy P, editors. Handbook of Mentalizing in Mental Health Practice. American Psychiatric Publishing, Inc.; 2012. p. 289-308.

(4) Suchman N, Pajulo M, Kalland M, DeCoste C, Mayes L. At-Risk Mothers of Infants and Toddlers. In: Bateman AW, Fonagy P, editors. Handbook of Mentalizing in Mental Health Practice. American Psychiatric Publishing, Inc.; 2012. p. 309-46. 
ORIGINALES Y REVISIONES

(5) Skarderud F, Fonagy P. Eating Disorders. In: Bateman AW, Fonagy P, editors. Handbook of Mentalizing in Mental Health Practice. American Psychiatric Publishing, Inc.; 2012. p. $347-84$

(6) Luyten P, Fonagy P, Lemma A, Target M. Depression. In: Bateman AW, Fonagy P, editors. Handbook of Mentalizing in Mental Health Practice. American Psychiatric Publishing, Inc.; 2012. p. 385-418.

(7) Allen JG, Lemma A, Fonagy P. Trauma. In: Bateman AW, Fonagy P, editors. Handbook of Mentalizing in Mental Health Practice. American Psychiatric Publishing, Inc.; 2012. p. 419-44.

(8) Philips B, Kahn U, Bateman AW. Drug Addiction. In: Bateman AW, Fonagy P, editors. Handbook of Mentalizing in Mental Health Practice. American Psychiatric Publishing, Inc.; 2012.p. $445-62$.

(9) Bleiberg E, Rossouw T, Fonagy P. Adolescent Breakdown and Emerging Borderline Personality Disorder. In: Bateman AW, Fonagy P, editors. Handbook of Mentalizing in Mental Health Practice. American Psychiatric Publishing, Inc.; 2012. p. 463-510.

(10) Bowlby J. Attachment and loss: volume 1. attachment. (Trad cast: El apego y la pérdida - 1. El apego. Barcelona: Paidós, 1998). New York: Basic Books; 1969.

(11) Bowlby J. Attachment and loss: volume 2. separation. (Trad cast: El apego y la pérdida - 2. La separación. Barcelona: Paidós, 1985). New York: Basic Books; 1973.

(12) Bowlby J. The making and breaking of affectional bonds (Trad cast: Vínculos afectivos: formación, desarrollo y pérdida, Madrid: Morata, 1986). London: Tavistock; 1979.

(13) Bowlby J. Attachment and loss: volume 3. loss. (Trad cast: El apego y la pérdida - 3. La pérdida. Barcelona: Paidós, 1993). New York: Basic Nooks; 1980.

(14) Bowlby J. El Vínculo afectivo (2ª reimpresión). Barcelona: Paidós; 1990.

(15) Fonagy P, Gergely G, Jurist E, Target M. Affect regulation, mentalization and the development of the self. New York: Other Press; 2002.

(16) Main M, Kaplan N, Cassidy J. Security in infancy, childhood and adulthood: a move to the level of representation. Monographs of the Society for Research in Child Development. 1985;50(1-2):66-104.

(17) Premack D, Woodruff G. Does the Chimpanzee Have a Theory of Mind. Behavioral and Brain Sciences. 1978;1(4):515-26.

(18) Baron-Cohen S, Leslie AM, Frith U. Does the autistic child have a "theory of mind"? Cognition. 1985;21:37-46.

(19) Baron-Cohen S. Mindblindness: an essay on autism and theory of mind. Boston: MIT Press/Bradford Books; 1995.

(20) Frith CD. The Cognitive Neuropsychology of Schizophrenia. Psychology Press; 1992.

(21) Frith C. Schizophrenia and theory of mind. Psychological Medicine. 2004;34:385-9.

(22) Bateman AW, Fonagy P. Handbook of Mentalizing in Mental Health Practice. Handbook of mentalizing in mental health practice. American Psychiatric Publishing, Inc.; 2012.

(23) Bateman AW, Fonagy P. Mentalization-based treatment of BPD. Journal of personality disorders. 2004; 18:36-51.

(24) Bateman AW, Fonagy P. Comorbid antisocial and borderline personality disorders: mentalization-based treatment. Journal of clinical psychology. 2008;64:181-94.

(25) Fonagy P, Bateman A. Mechanism of change in mentalization based treatment of borderline personality disorder. Journal of clinical Psychology. 2006;62:411-30. 
(26) Hesse E. The Adult Attachment Interview. Historical and current perspectives. In: Cassidy J, Shaver PR, editors. Handbook of attachment; theory, research and clinical applications. New York: Guilford; 1999. p. 395-433.

(27) Luyten P, Fonagy P, Lowyck B, Vermote R. Assesment of Mentalization. In: Bateman AW, Fonagy P, editors. Handbook of Mentalizing in Mental Health Practice. American Psychiatric Publishing, Inc.; 2012. p. 43-66.

(28) Fonagy P, Steele M, Moran G, Steele H, Higgit A. The capacity for understanding mental states: the reflective self in parent and child and its significance for security of attachment. Infant Mental Health Journal. 1991;13:201-18.

(29) Lanza Castelli G. Las polaridades de la Mentalización en la práctica clínica. Clínica e Investigación Relacional. 2011;5(2):295-315.

(30) Fonagy P, Moran GS, Edgcumbe R, Kennedy H, Target M. The roles of mental representations and mental processes in therapeutic action. The Psychoanalytic Study of the Child. 1993;48:9-48.

(31) Lanza Castelli G, Bilbao Bilbao I. Un método para la evaluación de la mentalización en el contexto interpersonal. Psychoanalytic Psychotherapy Review. European Federation for Psychoanalytic Psychotherapy. 2012;

(32) Fonagy P. Persistencias transgeneracionales del apego: una nueva teoría. Revista de Psicoanálisis [Internet]. 1999;3:1-17. Available from: http://www.aperturas.org/3fonagy.html;

(33) Fonagy P, Luyten P. A developmental, mentalization-based approach to the understanding and treatment of borderline personality disorder. Development and Psychopathology. 2009;21:1355-81.

(34) Fonagy P, Luyten P. Mentalization: Understanding Borderline Personality Disorder. In: Fuchs, Sattel, Henningsen, editors. The Embodied Self. Dimensions, Coherence and Disorders. Schattauer; 2010.

(35) Baron RA, Byrne D. Psicologia Social. Madrid: Prentice Hall; 1998.

(36) Penn DL, Sanna LJ, Roberts DL. Social cognition in schizophrenia: an overview. Schizophrenia Bulletin. 2008 May;34(3):408-11.

(37) Frith C, Corcoran R. Exploring "theory of mind" in people with schizophrenia. Psychological Medicine. 1996;26:521-30.

(38) Langdon R, Coltheart M. Mentalising , schizotypy , and schizophrenia. Cognition. 1999;71(1):43-71.

(39) Sarfati Y, Hardy-Baylé M, Besche C, D. W. Attribution of intentions to others in people with schizophrenia: a non-verbal exploration with comic strip. Schizophrenia Research. 1997;25:199209.

(40) Harrington L, Langdon R, Siegert RJ, McClure J. Schizophrenia, theory of mind, and persecutory delusions. Cognitive neuropsychiatry. 2005 Mar;10(2):87-104.

(41) Harrington L, Siegert RJ, McClure J. Theory of mind in schizophrenia: a critical review. Cognitive neuropsychiatry. 2005 Aug;10(4):249-86.

(42) Dziobek I, Fleck S, Kalbe E, Rogers K, Hassenstab J, Brand M, et al. Introducing MASC: a movie for the assessment of social cognition. Journal of autism and developmental disorders. 2006 Jul;36(5):623-36.

(43) Smeets T, Dziobek I, Wolf OT. Social cognition under stress: differential effects of stress-induced cortisol elevations in healthy young men and women. Hormones and behavior. Elsevier Inc.; 2009 Apr;55(4):507-13. 
ORIGINALES Y REVISIONES

(44) Salovey P, Grewal D. The science of emotional intelligence. Current Directions in Psychological Science. 2005;14(6):281-5.

(45) Baer RA, Smith GT, Allen KB. Assessment of mindfulness by self-report: The kentucky inventory of mindfulness skills. Assessment. 2004;11(3):191-206.

(46) Brown KW, Ryan RM. The benefits of being present: Mindfulness and its role in psychological well-being. Journal of Personality and Social Psychology. 2003;84:822-48.

(47) Lawrence EJ, Shaw P, Baker D, Baron-Cohen S, David AS. Measuring empathy: reliability and validity of the Empathy Quotient. Psychological Medicine. 2004 Jul;34(5):911-24.

(48) Fonagy P, Ghinai RA. A self-report measure of mentalizing development and preliminary test of the reability and validity of the Reflective Function Questionnaire (RFQ). Unpublished manuscript. London: University College; 2008.

(49) Luyten P, Mayes LC, Sadler L, Fonagy P, Nicholls S, Crowley M, et al. The Parental Reflective Functioning Questionnaire-1 (PRFQ-1). Unpublished manual. University of Leuven. Leuven, Belgium; 2009.

(50) Vrouva I, Fonagy P. Development of the Mentalizing Stories for Adolescents (MSA). Journal of the American Psychoanalytic Association. 2009;57(5):1174-8.

(51) Baron-Cohen S, Wheelwright S, Hill J, Raste Y, Plumb I. The "Reading the Mind in the Eyes" Test Revised Version: A Study with Normal Adults, and Adults with Asperger Syndrome or High-functioning Autism. Journal of child psychology and psychiatry, and allied disciplines. 2001 Mar;42(2):241-51.

(52) Slade A, Aber J, Berger B, Bresgi I, Kaplan M. The Parent Development Interview Revised. Unpublished manuscript, Yale Child Study Center, New Haven, CT.; 2002.

(53) Bouchard M, Target M, Lecours S, Fonagy P, Tremblay L, Schachter A, et al. Mentalization in adult attachment narratives: Reflective functioning, mental states, and affect elaboration compared. Psychoanalytic Psychology. 2008;25:47-66.

(54) Carcione A, Dimaggio G, Falcone M, Nicolo G, Procacci M, Semerari A. Metacognition Assessment Scale (MAS) 3.1. Rome: Centro di Psicoterapia Cognitiva: Unpublished; 2007.

(55) Beeghly M, Cicchetti D. Child maltreatment, attachment, and the self system: Emergence of an internal state lexicon in toddlers at high social risk. Development and Psychopathology. 1994;6:5-30.

(56) King-Casas B, Sharp C, Lomax-Bream L, Lohrenz T, Fonagy P, Montague P. The rupture and repair of cooperation in borderline personality disorder. Science. 2008;321:806-10.

(57) Beaumont R, Sofronoff K. A new computerised advanced theory of mind measure for children with Asperger syndrome: The ATOMIC. Journal of Autism and Developmental Disorders. 2008;38(2):249-60.

(58) Meins E, Fernyhough C. Mind-Mindedness Coding Manual. Unpublished manuscript, Durham University.; 2006.

(59) Cramer P. Storytelling, narrative, and the Thematic Apperception Test. New York: Guilford Press; 2004.

(60) Lanza Castelli G. El Test de Situaciones para la Evaluación de la Mentalización. Revista de la Asociación de Psicoterapia de la República Argentina. 2011;Año IV(No 2).

(61) Fonagy P, Target M, Steele H, Steele M. Reflective-functioning manual Version 5 for application to adult attachment interviews. London: Department of Clinical Health Psychology University College London; 1998. p. 1-47. 
(62) Extremera N, Fernández-Berrocal P, Salovey P. Spanish version of the Mayer-SaloveyCaruso Emotional Intelligence Test (MSCEIT). Version 2.0: reliabilities, age and gender differences. Psicothema. 2006 Jan; 18, Supl(2001):42-8.

(63) Lahera G, Boada L, Pousa E, Mirapeix I, Morón-Nozaleda G, Marinas L, et al. Movie for the Assessment of Social Cognition (MASC): Spanish Validation. Journal of autism and developmental disorders [Internet]. 2014 Feb 13 [cited 2014 May 9]; Available from: http://www.ncbi.nlm. nih.gov/pubmed/24522969

(64) Soler J, Tejedor R, Feliu-Soler A, Pascual JC, Cebolla A, Soriano J, et al. Psychometric proprieties of Spanish version of Mindful Attention Awareness Scale (MAAS). Actas españolas de psiquiatría. 2012;40(1):19-26.

(65) Pérez-Albéniz A, Paúl J De, Etxeberría J, Montes MP, Torres E. Adaptación de Interpersonal Reactivity Index ( IRI ) al español. Psicothema. 2003;15(2):267-72.

(66) Fernández-Abascal E, Cabello R, Fernández-Berrocal P, Baron-Cohen S. Test-retest reliability of the "Reading the Mind in the Eyes" test: a one-year follow-up study. Mol. Autism. 2013;33(4).

(67) Moral de la Rubia J, Retamales R. Estudio de validación de la Escala de Alexitimia de Toronto (TAS-20) en muestra española. Psiquiatria.com. Revista electrónica de Psicología. 2000;4:110 .

(68) Grienenberger JF, Kelly K, Slade A. Maternal reflective functioning, mother-infant affective communication, and infant attachment: exploring the link between mental states and observed caregiving behavior in the intergenerational transmission of attachment. Attachment \& human development. 2005 Sep;7(3):299-311.

(69) Shmueli-Goetz Y, Target M, Fonagy P, Datta A. The Child Attachment Interview: A Psychometric Study of Reliability and Discriminant Validity. Developmental Psychology. 2008 Jul;44(4):939-56.

(70) Bagby RM, Taylor GJ, Parker JDA, Dickens SE. The development of the Toronto Structured Interview for Alexithymia: item selection, factor structure, reliability and concurrent validity. Psychotherapy and Psychosomaticssychosomatics. 2006 Jan;75(1):25-39.

(71) Bagby RM, Parker JDA, Taylor GJ. The Twenty-Item Toronto Alesithymia Scale I. Item Selection and Cross-Validation of the factor structure. Journal of Psychosomatic Research. 1994;38(1):23-32.

(72) Lane RD, Quinlan DM, Schwartz GE, Walker PA, Zeitlin SB. The Levels of Emotional Awareness Scale. A Cognitive-Developmental Measure of Emotion. Journal of Personality Assessment. 1990;55(1\&2):124-34.

(73) Shill MA, Lumley MA. The Psychological Mindedness Scale: Factor structure, convergent validity and gender in a non-psychiatric sample. Psychology and Psychotherapy: Theory, Research and Practice. 2002;75:131-50.

(74) Rimes KA, Chalder T. The Beliefs about Emotions Scale: Validity, reliability and sensitivity to change. Journal of Psychosomatic Research. 2010;68(3):285-92.

(75) Brass M, Schmitt RM, Spengler S, Gergely G. Investigating action understanding: inferential processes versus action simulation. Current biology : CB. 2007 Dec;17(24):2117-21.

(76) Golan O, Baron-Cohen S, Hill JJ, Rutherford MD. The "Reading the Mind in the Voice" Test-Revised: A Study of Complex Emotion Recognition in Adults with and Without Autism Spectrum Conditions. Journal of Autism and Developmental Disorders. 2007 Jul;37(6):1096-106. 
(77) Tottenham N, Tanaka JW, Leon AC, McCarry T, Nurse M, Hare TA, et al. The NimStim set of facial expressions: Judgments from untrained research participants. Psychiatry Research. 2009;168(3):242-9.

(78) Lieberman MD, Eisenberger NI, Crockett MJ, Tom SM, Pfeifer JH, Way BM. Putting Feelings Into Words. Affect Labeling Disrupts Amygdala Activity in Response to Affective Stimuli. Psychological Science. 2007;18(5):421-8.

(79) Golan O, Baron-Cohen S, Hill JJ, Golan Y. The "Reading the Mind in Films" Task: Complex emotion recognition in adults with and without autism spectrum conditions. Social Neuroscience. $2006 \mathrm{Jan} ; 1(2): 111-23$.

(80) Golan O, Baron-Cohen S, Hill J. The Cambridge Mindreading (CAM) Face-Voice Battery: Testing complex emotion recognition in adults with and without Asperger syndrome. J Autism Dev Disord. 2006;36(2):169-183.

(81) Sonnby-Borgström M, Jönsson P. Dismissing-avoidant pattern of attachment and mimicry reactions at different levels of information processing. Scandinavian journal of psychology. 2004 Apr;45(2):103-13.

(82) Pichon S, de Gelder B, Grèzes J. Two different faces of threat. Comparing the neural systems for recognizing fear and anger in dynamic body expressions. Neuroimage. 2009;47(4):187383 .

(83) Bailey CA, Pendl J, Levin A, Olsen S, Langlois E, Crowley MJ, et al. Yale Child Study Center. Face Morphing Tutorial : From Models to Morphs. New Haven: Yale University; 2008.

(84) Hein G, Singer T. I feel how you feel but not always: the empathic brain and its modulation. Current opinion in neurobiology. $2008 \mathrm{Apr}$;18(2):153-8.

(85) Blackshaw A, Kinderman P, Hare D, Hatton C. Theory of mind, causal attribution and paranoia in Asperger syndrome. Autism. 2001;5(2):147-63.

(86) Diamond D, Blatt S, Stayner D, Kaslow N. Self-other differentiation of object representations. Unpublished manuscript: Yale University at New Haven; 1991. 